\title{
Preface to the Special Issue on Process Querying and Declarative, Decision and Hybrid Approaches to Processes 2019
}

\author{
Claudio Di Ciccio ${ }^{1} \cdot$ Søren Debois ${ }^{2} \cdot$ Artem Polyvyanyy $^{3} \cdot$ Tijs Slaats $^{4} \cdot$ Arthur ter Hofstede $^{5} \cdot$ Jan Vanthienen $^{6}$
}

Published online: 27 May 2021

(c) The Author(s), under exclusive licence to Springer-Verlag GmbH Germany, part of Springer Nature 2021

Business processes involve rules and decisions describing the premises and possible outcomes of specific situations. However, rules and decisions are often implicit in process flows, process activities or in the head of employees (tacit knowledge). To make them explicit, they require the employment of techniques capable of capturing behavioural constraints and factors determining decisions. Process querying studies automated methods, techniques, and tools for filtering, retrieving, and updating models that describe observed or envisioned processes, and relationships between these processes with the ultimate goal of converting process-related information into decision-making capabilities.

This Special Issue of the Journal on Data Semantics focuses on the interplay between process querying and declarative approaches to processes. It includes the extended versions of selected papers from the International Workshop on Declarative, Decision and Hybrid approaches to Processes (DEC2H) 2019 and the International Workshop on Process Querying (PQ) 2019, both co-located with the

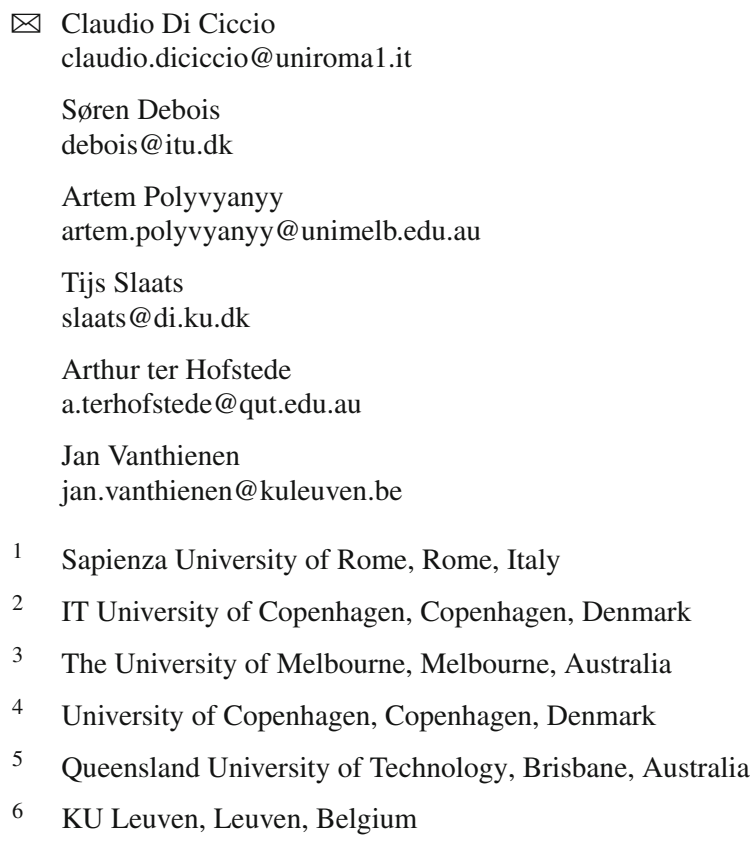

Business Process Management Conference (BPM) 2019 in Vienna (Austria). The invited papers were selected based on their quality, significance of their results, and relevance to the themes of the workshops and the journal. All papers went through an additional peer-review process.

Throughout their paper, "Multi-Dimensional Event Data in Graph Databases", Esser and Fahland propose a novel approach to store and query multi-dimensional event data in graph-based data structures. The authors highlight the suitability of the approach for multi-dimensional process mining, in which events stem from different sources yet pertain to correlated processes.

In their paper entitled "Formal Framework for Checking Compliance of Data-Driven Case Management", Haarmann, Holfter, Pufahl and Weske present a hybrid case-management approach for process modelling at the crossroads of activitycentric and data-centric paradigms. They give a formal semantics rooted into Petri nets and then use model checking for Petri nets to statically verify compliance properties.

Finally, the paper entitled "Integrating BPMN and DMNModeling and Analysis" by de Leoni, Felli and Montali, builds upon the themes illustrated in the keynote of DEC2H 2019. In particular, the authors introduce a formal model to manage the interplay of control-flow, data and decision aspects in the representation of process behaviour. They define the execution semantics based on Data Petri nets and illustrate the suitability of their formalism for automated verification.

The contributions to this Special Issue highlight the growing interest of research in techniques for the specification, querying and checking of processes that operate on multiple perspectives and span across different modelling paradigms. These articles illustrate the advantages of such holistic approaches and pave the way for future endeavours in the development of new techniques that further broaden the spectrum of process analytics.

Publisher's Note Springer Nature remains neutral with regard to jurisdictional claims in published maps and institutional affiliations. 